\title{
Complicações fetais em gestações tardias
}

\author{
Fetal complications in late pregnancies
}

\section{Complicaciones fetales en gestaciones tardias}

\author{
Thayro Van Der Maas do Bem, Thiago S. Sampaio, \\ Denise L. M. Monteiro," Danielle B. S. Barmpas
}

\section{Resumo}

Em virtude de mudanças sociais e no estilo de vida, a incidência de gestação tardia, em mulheres com mais de 35 anos, aumentou significativamente nas últimas décadas. Tal aumento contribuiu para taxas mais altas de complicações obstétricas e fetais. Foi realizada uma revisão crítica da literatura com o objetivo de avaliar a evidência disponível sobre a frequência das principais complicações gestacionais em gestações tardias, assim como possíveis explicações e controvérsias sobre o tema. A busca detalhada da literatura foi realizada incluindo as bases de dados: Medline (pelo PubMed), SciELO e LILACS. Os desfechos gestacionais e perinatais avaliados foram: idade gestacional no parto, baixo peso ao nascer (BPN $<2,5 \mathrm{~kg}$ ), índice de Apgar $<7$ no $1^{0}$ e no $5^{\circ}$ minutos, aumento na incidência de cesarianas e natimortalidade. Os resultados obtidos foram: idade gestacional no parto (<37 sem = de 3,4 a 51,4\%; > $42 \mathrm{sem}=$ de 0,4 a 6,9\%), natimortalidade (de zero a 3,2\%), BPN (de zero a $26,8 \%$ ), Apgar $<7\left(1^{\circ}\right.$ minuto $=$ de 5,6 a 22,9\%; $5^{\circ}$ minuto $=$ de 0,9 a 5\%), taxa de cesarianas (de 31,9 a 72,4\%). 0 impacto relativo ao aumento da idade materna e doenças crônicas nos desfechos gestacionais requer mais pesquisas. Profissionais de saúde devem estar em alerta para as particularidades das gestações tardias a fim de identificar potenciais complicações a tempo. Uma equipe multiprofissional especializada deve estar disponível para garantir as necessidades específicas em termos de exames e internações. Desta forma, as gestações tardias podem ser conduzidas de maneira mais segura, reduzindo taxas de complicações e desfechos adversos maternos e perinatais.

Descritores: Idade materna; Lactente; Baixo peso ao nascer; Índice de Apgar; Natimortalidade; Complicações na gravidez.

\begin{abstract}
In view of social and lifestyle changes, the incidence of advanced maternal age pregnancy, i.e. in women over 35 years, has markedly increased in recent decades. Such surge contributed to higher rates of obstetric and fetal complications. A critical review of the literature was conducted with the objective of assessing the available evidence on the frequency of the main pregnancy complications in advanced maternal age as well as possible explanations and controversies about the matter. A detailed search of medical literature was performed including the databases: Medline (via PubMed), SciELO and LILACS. Gestational and perinatal outcomes assessed were: gestational age at delivery, low birth weight (LBW $<2.5 \mathrm{~kg}$ ), Apgar score $<7$ at 1 and 5 minutes, increase in the incidence of cesarean sections and stillbirth. The results retrieved were: gestational age $(<37$ weeks $=3.4$ to $51.4 \%$; $>42$ weeks $=0.4$ to $6.9 \%$ ), stillbirth ( 0 to $3.2 \%$ ), LBW ( 0 to $26.8 \%$ ), Apgar score $<7$ (15t $\mathrm{min}=5.6$ to $22.9 ; 5^{\text {th }}$ min $=0.9$ to $5 \%$ ), cesarean section (31.9 to $72.4 \%$ ). The relative impact of maternal ageing and chronic diseases on pregnancy outcomes requires further research. Health professionals must be alert to the peculiarities of advanced maternal age pregnancy in order to timely identify potential complications. A specialized multiprofessional team should be available to warrant specific needs regarding exams and admission. Thus, advanced maternal age pregnancy can be more safely managed, reducing the rate of complications and maternal and perinatal adverse outcomes.
\end{abstract}

Keywords: Maternal age; Infant; Low birth weight; Apgar score; Stillbirth; Pregnancy complications. 


\section{Resumen}

El objetivo de este estudio es revisar la literatura en busca de informaciones que permitan identificar las principales complicaciones fetales de las gestaciones tardías (edad materna superior a 35 años de edad), filtrar las eventuales pruebas de su ocurrencia y/o confrontar supuestas divergencias en la prevalencia. Se realizó una amplia investigación en la literatura médica buscando identificar y extraer informaciones de la literatura nacional e internacional por medio de las siguientes bases de datos: Medline (por medio del PubMed), Scielo y LILACS. La incidencia del embarazo tardío viene aumentando significativamente en las últimas décadas en todo el mundo. Ese incremento concurre con altas tasas de complicaciones maternas y fetales. La mayor frecuencia de enfermedades adquiridas con la edad como artritis, hipertensión arterial crónica, diabetes, depresión, cancer y enfermedades cardíacas contribuye al mayor riesgo obstétrico, acarreando repercusiones fetales. Para expresar las complicaciones fetales fueron definidas las siguientes variables de estudio: edad gestacional, bajo peso al nacer, Apgar $<7$ en el $1^{\circ}$ y $5^{\circ}$ minutos, aumento del número de cesáreas y mortalidad al nacer. El estudio mostró los siguientes resultados: edad gestacional ( $<37$ semanas $=3,4$ a 51,4 \%; $>42$ semanas $=0,4$ a 6,9\%), mortalidad al nacer (cero a 3,2\%), BPN (cero a 26,8\%), Apgar $<7\left(1^{\circ}\right.$ minuto $=5,6$ a 22,9\%; $5^{\circ}$ minuto $=0,9$ a $5 \%$ ), tasa de cesárea (31,9 a 72,4\%). La relativa contribución del aumento de la edad materna y de las enfermedades crónicas en los resultados gestacionales necesita de más estudios. Los profesionales de salud deben estar atentos en las características del embarazo en esta fase de la vida, apuntando a identificar precozmente posibles complicaciones, debiendo contar con servicio especializado que garantice la tramitación para la internación y realización de exámenes específicos en los casos necesarios. De esta manera, esta gestación podrá ser acompañada con más seguridad, reduciendo la posibilidad de complicaciones para el binomio materno-fetal.

Palabras clave: Edad materna; Lactente; Bajo peso al nacer; Índice de Apgar; Mortalidad al nacer; Complicaciones gestacionales.

\section{Introdução}

A ocorrência de gravidez em mulheres com idade superior a 35 anos é denominada gravidez tardia. Sua frequência vem aumentando consideravelmente, fato relatado em estudos brasileiros e internacionais. ${ }^{1-5}$

No Brasil, entre 2003 e 2012, o número de mulheres que engravidaram entre os 40 e 44 anos passou de 53.016 para 62.371 , traduzindo crescimento de 17,6\%. A gravidez entre 35 e 39 anos aumentou $26,3 \%$ neste período (201.077 para 254.011 gestações). ${ }^{6}$

De 1970 a 2000, nos Estados Unidos, o nascimento de filhos de mulheres com 35 anos ou mais subiu de 5\% para 13\%. ${ }^{7}$ Em 2009, atingiu 14,2\%, com crescimento de $3 \%$ em idades de 40 a 44 anos, demonstrando tendência que se repete em outras nações, sobretudo nos países industrializados. ${ }^{7,8}$

Os motivos para postergar a gravidez variam, incluindo o desejo das mulheres de investir na carreira educacional e profissional, adiar o casamento, casar de novo, ampla disponibilidade de métodos anticoncepcionais e problemas relacionados à infertilidade. ${ }^{1} \mathrm{O}$ fato é que em comparação com mulheres mais jovens, aquelas com mais de 35 anos experimentam mais abortos espontâneos ou induzidos, maior risco de morte perinatal, de baixa vitalidade e baixo peso do recém-nascido. A gravidez tardia também se associa a complicações fetais como: anomalias cromossômicas, sofrimento fetal, internação em UTI, restrição do crescimento fetal, além de taxa de perda fetal tardia inexplicável. ${ }^{1-3,8,9}$

O maior risco obstétrico em gestantes de idade avançada pode ser decorrente da frequência aumentada de doenças crônicas, especialmente hipertensão arterial, diabetes mellitus e mioma uterino..$^{10,11}$ No entanto, como estas doenças podem constituir-se em variáveis de confusão, a avaliação de riscos associados à idade materna isoladamente pode ser prejudicada., ${ }^{5,12}$ Os resultados adversos podem, em parte, estar relacionados ao aumento da morbidade e complicações obstétricas durante a gravidez e o parto. ${ }^{3}$ Os riscos são elevados mesmo para uma mulher saudável, sobretudo em relação à prematuridade e ao aumento na frequência de cesárea. ${ }^{5,12-14}$

O número crescente de gravidezes em idade tardia justifica a investigação do prejuízo possível ao feto e suas consequências no desenvolvimento. Sendo assim, esse trabalho tem o objetivo de revisar a literatura para identificar as principais complicações fetais das gestações tardias, filtrar as eventuais justificativas de seu acontecimento e/ou confrontar supostas divergências de pre- 
Quadro 1. Critérios de inclusão e exclusão na revisão crítica sobre complicações fetais de gestações em idade tardia.

\begin{tabular}{|l|l|}
\hline \multicolumn{1}{|c|}{ Critérios de inclusão } & \multicolumn{1}{c|}{ Critérios de exclusão } \\
\hline $\begin{array}{l}\text { 1. Foco em complicações fetais de gestantes com idade } \\
\text { entre 35 e 50 anos; }\end{array}$ & 1. Complicações maternas e gemelaridade; \\
$\begin{array}{l}\text { 2. Estudo limitado a gestações espontâneas; } \\
\begin{array}{l}\text { 3. Estudos que relacionam complicações fetais com idade } \\
\text { avançada; }\end{array}\end{array}$ & 2. Estudos sobre gestação por fertilização assistida; \\
$\begin{array}{l}\text { 4. Estudos em Português, Inglês, Espanhol e Francês. } \\
\end{array}$ & 4. Estudos anteriores a 2008. \\
\hline
\end{tabular}

\section{Quadro 2.}

Variáveis estabelecidas ao parâmetro de prejuízo ao feto.
A - baixo peso ao nascer (BPN)
B - idade gestacional ao nascer
C - tipo de parto
D - Apgar no 1ㅇ e 50 minuto
E - natimortalidade

valência.

\section{Métodos}

Foi realizada uma revisão crítica com base em pesquisa bibliográfica na mídia digital nacional e internacional. Para a pesquisa do Medline (por meio do Pubmed), utilizou-se a seguinte estratégia de busca: "Maternal Age"[Majr] e "Pregnancy Complications"[Mesh], tendo sido encontrados 150 artigos. Para a pesquisa realizada nas bases de dados Lilacs e SciELO, foram utilizados os termos "advanced maternal age" e "idade materna avançada", totalizando 49 artigos. Foram selecionados artigos publicados a partir de 2008, que apresentassem dados epidemiológicos e recomendações especificamente relacionadas ao tema, obedecendo-se aos critérios de inclusão e exclusão pré-estabelecidos (Quadro 1) e que apresentassem pelo menos uma das variáveis definidas como parâmetro de prejuízo ao feto (Quadro 2).

Essa seleção resultou em 20 artigos, dos quais 11 foram utilizados para formular os resultados. Os artigos restantes forneceram dados para enriquecer a introdução e a discussão.

O detalhamento da forma de seleção dos artigos utilizados na confecção deste estudo encontra-se no fluxograma (Figura 1).

\section{Resultados}

Foram utilizados 11 estudos que incluíam as variáveis de interesse para se proceder a análise dos resultados. A descrição do desenho de cada um deles, assim como os resultados da idade gestacional, natimortalidade, baixo peso ao nascer, índice de Apgar $<7$ no primeiro e quinto minuto e taxa de cesárea, encontra-se no quadro 1.

A prematuridade ( $\mathrm{IG}<37$ semanas) foi avaliada em todos os 11 estudos, variando entre 3,4 e $51,4 \%$, enquanto a IG $>42$ semanas foi analisada em cinco estudos, mostrando valores entre 0,4 e 6,9\%. O BPN foi referido em 10 dos 11 estudos analisados, com índices entre 0 e $26,8 \%$. O índice de Apgar menor que 7 no $1^{\circ}$ minuto foi avaliado em 5 estudos variando de 5,6 a 22,9\%, enquanto o índice inferior a 7 no $5^{\circ}$ minuto variou de $0,9 \%$ a 5,0\%. A natimortalidade foi avaliada em 8 estudos, variando de 0 a 3,2\%. A taxa de cesáreas mostrou-se heterogênea, sendo descrita em 8 estudos, variando de 31,9 a $72,4 \%$.

\section{Discussão}

Pesquisas mostram que mulheres com idade superior a 35 anos apresentam maior frequência de resultados perinatais adversos, com destaque para: $\mathrm{RN}$ com baixo peso, prematuro, índice de Apgar menor que 7 no $1^{\circ}$ e $5^{0}$ minutos de vida, maior número de partos operatórios, além de óbitos fetais (Quadro 1). . $^{1-3,9-1,15-19}$

$\mathrm{O}$ aumento da idade materna mostra associa- 


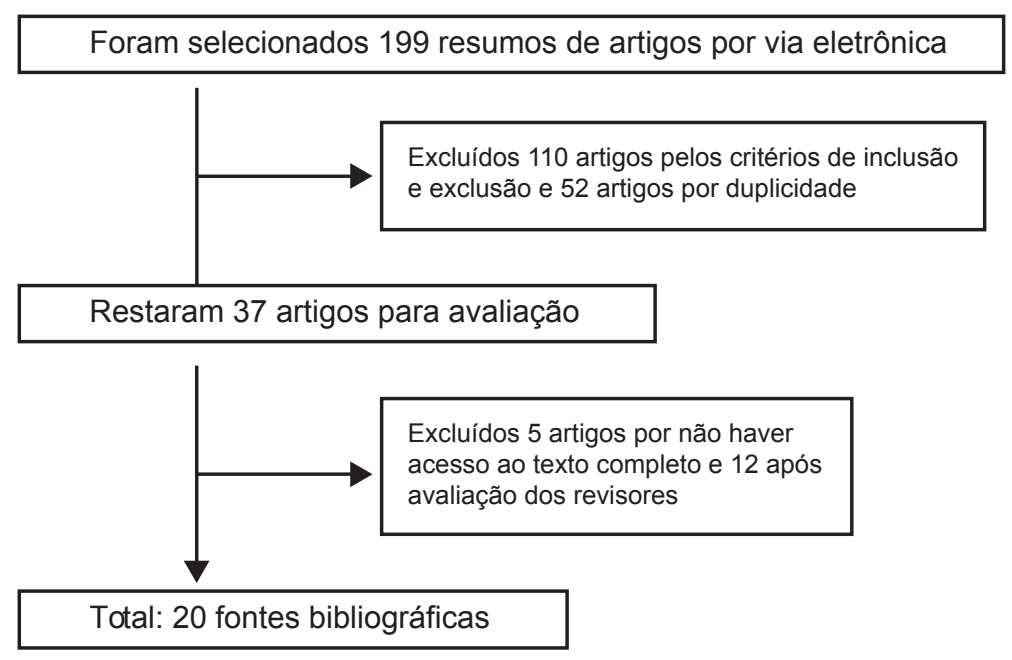

Figura 1. Fluxograma de busca de referências

ção com resultados obstétricos adversos, sugerindo que o risco aumenta proporcionalmente à idade da mãe. Em mulheres acima de 40 anos, o risco de mortalidade perinatal foi 2,2 vezes maior em comparação com gestantes com menos de 35 anos. ${ }^{7}$ Salem e colaboradores encontraram significante associação entre mortalidade perinatal e aumento da idade materna, o que não foi confirmado ao controlar com possíveis variáveis de confusão como crescimento intrauterino restrito e malformações congênitas. ${ }^{17}$ Entre os estudos pesquisados nesta revisão, $45 \%$ não descreveram a taxa de natimortalidade, o que pode ser considerado uma das limitações do estudo. 2,3,9,11,19

O BPN pode estar relacionado com aumento na taxa de mortalidade perinatal. Lisonkova e colaboradores encontraram taxas de BPN e de mortalidade fetal e perinatal significativamente maiores entre as mães com maior idade, respectivamente $66 \%$ e $26 \% .{ }^{1 "}$ Da mesma forma, Salem e colaboradores descreveram alta taxa de BPN coincidente com maior taxa de mortalidade fetal, sugerindo relação entre os dois fatos, já que a alta prevalência de BPN na gravidez tardia pode estar relacionada a doenças adquiridas com a idade como: artrite, hipertensão arterial crônica, depressão, câncer e infarto agudo do miocárdio. ${ }^{10,11,17}$

A comparação entre as idades gestacionais evidenciou que tanto a prematuridade quanto o nascimento pós-termo estiveram presentes entre as mulheres em idade avançada, ${ }^{1}$ sendo mais marcante a baixa idade gestacional. No entanto, a taxa de prematuridade mostrou-se extremamente variável, dificultando mais conclusões.,15 Essa variação pode ser resultado do nível sociocultural de cada local, além da maior frequência de casos de placenta prévia, acretismo placentário, descolamento da placenta e distúrbios hipertensivos em grávidas de idade avançada, o que pode gerar viés de confusão..$^{3,10,16,18} \mathrm{O}$ desenvolvimento dessas complicações pode estar relacionado à vasculopatia úteroplacentária. Hsieh e colaboradores evidenciaram que a taxa de lesões por esclerose nas artérias do miométrio aumentou com a idade, de $11 \%$ entre $17-19$ anos para $61 \%$ entre $30-39$ anos e para $83 \%$ após 39 anos. $^{18}$

O índice de Apgar é um bom indicador do resultado perinatal, sendo relevante para prognóstico do nascimento. As gestantes em idade tardia apresentam 5,7 vezes mais chance de ter filhos com índice de Apgar inferior a 7 no $5^{\circ} \mathrm{minu}-$ to de vida do que as mais jovens. ${ }^{1}$ No entanto, os resultados desta revisão mostram que a maioria dos recém-natos que apresentavam Apgar menor que 7 no $1^{0}$ minuto recuperaram o índice no $5^{0}$ minuto de vida. ${ }^{1,10,15,18}$

O índice de cesáreas na gravidez tardia mostrou-se superior a 50\% na maioria dos estudos. Entre as várias razões que levam à alta taxa de cesáreas, a ineficiência do miométrio envelhecido, a diminuição do número de receptores de ocitocina e a distocia estão entre as principais causas. ${ }^{1,20}$ No entanto, no Brasil é comum a ocorrência de maior índice de cesariana em grupos de menor risco obstétrico, como as mulheres de status sociais mais elevados, independentemente de critérios 
técnicos e obstétricos. Dessa forma, a avaliação desse dado pode estar prejudicada, sendo outra limitação do estudo.

O parto cesáreo pode contribuir para aumentar a morbidade materna, além de elevar o risco de mortalidade neonatal, bem como favorecer complicações placentárias em gravidezes posteriores, incluindo a placenta prévia e descolamento prematuro da placenta.'

As gestantes com 35 anos ou mais apresentam risco 1,5 vezes maior de complicações na gravidez e de 1,6 a 2,6 maior para resultados perinatais adversos quando comparadas a mulheres de 20 a 34 anos. Após ajuste para variáveis de confundimento, entre as de 35 a 39 anos permaneceu o maior risco de partos operatórios vaginais $(\mathrm{OR}=1,5 ; \mathrm{IC} 95 \%$ 1,2 a 1,7) e de cesáreas $(\mathrm{OR}=1,6$; IC95\% 1,5 a 1,7). Entre as mulheres com 40 anos ou mais foi confirmado maior risco de parto prematuro ( $\mathrm{OR}=1,7$; IC95\% 1,3 a 2,2), parto operatório vaginal $(\mathrm{OR}=3,1 ; \mathrm{IC} 95 \%$ 2,0 a 4,6) e parto cesáreo $\left(\mathrm{OR}=2,6 ; \mathrm{IC} 95 \%\right.$ 2,2 a 3,1 )..$^{18}$

A natimortalidade é tida como multifatorial e, embora a gravidez em idade tardia contribua para o risco de morte fetal em países desenvolvidos, outros fatores - como infecção, desnutrição, falta de cuidados pré-natais e pobreza - são os principais responsáveis nos países em desenvolvimento. O mecanismo biológico do aumento de morte fetal com a idade materna avançada é incerto, mas pode ser atribuído a alguns fatores, como doenças crônicas e complicações médicas ou obstétricas, ${ }^{1,4}$ sendo que mulheres mais velhas têm maior risco de sofrer hipertensão induzida pela gravidez ou diabetes gestacional. $^{18}$ Soma-se o já descrito efeito direto do envelhecimento materno, que está relacionado com a deficiência de perfusão placentária causada pela baixa vascularização uterina.'

$\mathrm{O}$ aumento da idade mostrou associação significativa com aborto para mulheres de 35-39 anos e com 40 ou mais ( $\mathrm{OR}=2,0$ e 2,4, respectivamente), anormalidades cromossômicas ( $\mathrm{OR}=4,0$ e 9,9), anomalias congênitas ( $O R=1,4$ e 1,7), diabetes gestacional $(\mathrm{OR}=1,8$ e 2,4), placenta prévia $\left(\mathrm{OR}=1,8\right.$ e 2,8) e taxa de cesarianas $\left(\mathrm{OR}=1,6\right.$ e 2,0). ${ }^{7}$ No entanto, a relativa contribuição do aumento da idade materna e das doenças crônicas nos resultados gestacionais necessita de mais estudos. ${ }^{4}$

\section{Considerações finais}

A gravidez em idade tardia tende a aumentar gradativamente em todo o mundo. Assim, as mulheres devem ser alertadas por seus médicos sobre os riscos da gravidez tardia, considerando inclusive que, com o aumento da idade materna, eleva-se a prevalência de comorbidades, responsáveis por gerar altas taxas de complicações fetais.

Os profissionais de saúde devem dedicar atenção especial a este grupo etário, estando alertas para as características da gravidez nesta fase da vida, visando identificar precocemente possíveis alterações. Ademais, é importante contar com serviço especializado que garanta o encaminhamento para internação e a realização de exames específicos nos casos necessários. Desta maneira, essa gestação poderá ser acompanhada com mais segurança, reduzindo a possibilidade de complicações para o binômio materno-fetal.

\section{Referências}

1. Gravena AAF, Sass A, Marcon SS, Pelloso SM. Outcomes in late-age pregnancies. Rev Esc Enferm USP. 2012;46(1):15-21. [periódico na Internet]. 2012 Feb [Citado em 03 de jun 2014]; 46(1): 15-21. Disponível em: http://www.scielo. br/scielo.php?script $=$ sci_arttext\&pid $=$ S008062342012000100002\&lng=en. http://dx.doi. org/10.1590/S0080-62342012000100002

2. Kenny LC, Lavender T, McNamee R, O’Neill SM, Mills T, Khashan AS. Advanced maternal age and adverse pregnancy outcome: evidence from a large contemporary cohort. PLoS One. 2013;8(2):e56583. http://dx.doi.org/10.1371/journal.pone.0056583

3. Delpisheh A, Brabin L, Attia E, Brabin BJ. Pregnancy late in life: a hospital-based study of birth outcomes. J Womens Health (Larchmt). 2008;17(6):965-70. http://dx.doi.org/10.1089/jwh.2008.0514

4. Huang L, Sauve R, Birkett N, Fergusson D, van Walraven C. Maternal age and risk of stillbirth: a systematic review. CMAJ. 2008;178(2):165-72. http:// dx.doi.org/10.1503/cmaj.070150

5. Andrade PC, Linhares JJ, Martinelli S, Antonini M, Lippi UG, Baracat FF. Resultados perinatais em grávidas com mais de 35 anos: estudo controlado. Rev Bras Ginecol Obstet. 2004;26(9):697-702. http:// dx.doi.org/10.1590/S0100-72032004000900004

6. IBGE. Instituto Brasileiro de Geografia e Estatística. Brasil vê crescimento de gravidez após os 40 anos. Disponível em: http://www.diariodepernambuco. 
com.br/ app/noticia/ciencia-e-saude/2014/02/10/ internas_cienciaesaude. Acesso em 15 Fev 2014.

7. Cleary-Goldman J, Malone FD, Vidaver J, Ball $\mathrm{RH}$, Nyberg DA, Comstock $\mathrm{CH}$, et al. Impact of maternal age on obstetric outcome. Obstet Gynecol. 2005;105(5 Pt 1):983-90.

8. Martin JA, Hamilton BE, Ventura SJ, Osterman MJK, Kirmeyer S, Mathews TJ, Wilson EC. Births: Final data for 2009. Division of Vital Statistics National Vital Statistics Reports. 201; 60(1):1-72. 3 nov 2011

9. Bakker R, Steegers EAP, Biharie AA, Mackenbach JP, Hofman A, Jaddoe VWV. Explaining differences in birth outcomes in relation to maternal age: the Generation R study. BJOG. 2011;118(4):500-9. http:// dx.doi.org/10.1111/j.1471-0528.2010.02823.x

10. Tabcharoen C, Pinjaroen S, Suwanrath C, Krisanapan O. Pregnancy outcome after age 40 and risk of low birth weight. J Obstet Gynaecol. 2009;29(5):378-83. http://dx.doi.org/10.1080/01443610902929537

11. Lisonkova S, Paré E, Joseph KS. Does advanced maternal age confer a survival advantage to infants born at early gestation? BMC Pregnancy Childbirth. 2013;13:87. http://dx.doi.org/10.1186/1471-2393-13-87.

12. Chan BC, Lao TT. Effect of parity and advanced maternal age on obstetric outcome. Int J Gynaecol Obstet. 2008;102(63):237-41. http://dx.doi. org/10.1016/j.ijgo.2008.05.004

13. Jolly M, Sebire N, Harris J, Robinson S, Regan L. The risks associated with pregnancy in women aged 35 years or older. Hum Reprod. 2000;15(11):2433-7.

14. Azevedo GD, Freitas Júnior RAO, Freitas AKMSO, Araújo ACPF, Soares EMM, Maranhão TMO. Efeito da idade materna sobre os resultados perinatais. Ver Bras Ginecol Obstet. 2002;24(3):181-5.

15. Sass A, Gravena AAF, Pelloso SM, Marcon SS. Resultados perinatais nos extremos da vida reprodutiva e fatores associados ao baixo peso ao nascer. Rev Gaúcha Enferm. 2011;32(2):352-8.

16. Ojule JD, Ibe VC, Fiebai PO. Resultado da gravidez em primigestas idosas. Ann Afr Med. 2011;10:204-8.
17. Salem YS, Levy A, Wiznitzer A, Holcberg G, Mazor $M$, Sheiner E. A significant linear association exists between advanced maternal age and adverse perinatal outcome. Arch Gynecol Obstet. 2011;283(4):755-9. http://dx.doi.org/10.1007/s00404-010-1459-4

18. Hsieh TT, Liou JD, Hsu JJ, Lo LM, Chen SF, Hung TH. Advanced maternal age and adverse perinatal outcomes in an Asian population. Eur J Obstet Gynecol Reprod Biol. 2010;148(1):21-6. http://dx.doi. org/10.1016/j.ejogrb.2009.08.022

19. Gitau GM, Liversedge H, Goffey D, Hawton A, Liversedge N, Taylor M. The influence of maternal age on the outcomes of pregnancies complicated by bleeding at less than 12 weeks. Acta Obstet Gynecol Scand. 2009;88(1):116-8.

20. Bayrampour H, Heaman M. Advanced maternal age and the risk of cesarean birth: a systematic review. Birth. 2010;37(3):219-26.

\section{Thayro Van Der Maas do Bem}

Disciplina de Obstetrícia. Departamento Ginecologia/ Obstetrícia. Centro Universitário Serra dos Órgãos (UNIFESO). Teresópolis, RJ, Brasil.

\section{Thiago S. Sampaio}

Disciplina de Obstetrícia. Departamento Ginecologia/ Obstetrícia. Centro Universitário Serra dos Órgãos (UNIFESO). Teresópolis, RJ, Brasil.

\section{Denise L. M. Monteiro}

Disciplina de Obstetrícia. Departamento de Ginecologia e Obstetrícia. Centro Universitário Serra dos Órgãos (UNIFESO). Teresópolis, RJ, Brasil.

Disciplina de Obstetrícia. Departamento de Ginecologia e Obstetrícia. Faculdade de Ciências Médicas da Universidade do Estado do Rio de Janeiro. Rio de Janeiro, RJ, Brasil.

\section{Danielle B. S. Barmpas}

Programa de Pós-graduação em Ciências Médicas. Faculdade de Ciências Médicas. Universidade do Estado do Rio de Janeiro. Rio de Janeiro, RJ, Brasil. 